\title{
Exploring Humanoid Factors of Robots through Transparent and Reflective Interactions
}

\author{
Shuo Niu, D. Scott McCrickard, Steve Harrison \\ Department of Computer Science \\ Virginia Tech \\ Blacksburg, VA 24060 \\ \{shuoniu, mccricks, srh\}@cs.vt.edu
}

\begin{abstract}
Humanoid robots that resemble human body, language and/or actions enable promising interactive agent behavior in people's life. The humanoid factor of being "like-a-human" implies that the interaction with a humanoid robot shares similarities with human-tohuman communications. Designing a humanoid robot must take the users' perception of its social characteristics into consideration to achieve intuitive and smooth interaction. The addition of humanoid factors means that traditional HCI knowledge need to be revisited and revised as it is applied to humanoid robot design. This work compares and contrasts the interaction paradigms for humanoid robots to traditional computer-based systems. Opportunities and challenges brought by humanoid factors are explored and discussed within two complementary aspects of human-computer interaction: transparent interaction and reflective interaction. Given the diverse roles of robots that are probable in future daily life, the impacts of humanoid factors in human-robot collaboration are also investigated to identify methods to optimize the mutual understanding between a human user and the robot. Grounded in recent robot research, this paper aims to enhance the knowledge of humanoid factors utilization and elucidate the shifting of interaction paradigms from human-computer interaction to humanrobot interaction.
\end{abstract}

Keywords- HRI, HCI, humanoid, transparent interaction, reflective interaction, collaboration

\section{INTRODUCTION}

Creating self-operating, human-like machines and automation to reduce the labor and mental work of people has been a dream of mankind for centuries. Modern robots appeared in the 1940s in many industrial and commercial departments for labor-intensive and hazardous tasks like assembly and exploration. Advancement in artificial intelligence and automation technologies enable contemporary robots to interact and collaborate directly with humans in many situations, such as household tasks, education, health care and emergency rescue. With the emerging ubiquity of robots in daily life, it becomes important to understand the users' perceptions to the interaction with a robot to expand the knowledge in user-friendly robot design and implementation.

Humanoid factors, characterized by resemblance to humans entirely or partially in appearance (and perhaps imitating human behaviors) is an important feature of robots and androids. As imagined in many science fiction movies, future robots will not be a machine without feelings and moods - they will be able to think and behave as a human, and interact physically and emotionally with humans. Compared to traditional human-computer interaction (HCI), which historically involves input and output devices such as keyboards, mice, and screens, the humanoid features of a robot create unique interaction experiences in which users may feel like they are communicating with a living creature - perhaps even a real human - instead of a machine. In many cases, the mental and physical behavioral habits found in social interactions (e.g. nodding the head to support, hugging to show intimacy) might be adopted to the interaction with a humanoid robot. These approaches can be utilized to create natural interaction paradigms and enhance the human-robot interaction experience.

Human-robot interaction (HRI) opens a new gateway to traditional HCI theories. Transparency and reflectivity, which are recognized as a pair of key counterparts of interaction in contemporary HCI theory, both potentially can be improved by properly configured humanoid factors [1]. However, humanoid factors also introduce challenges to traditional usability engineering, as the complexity and subtleness of the human social behaviors might be brought into the human-robot interaction. This requires the interaction designers to reexamine previous HCI knowledge and practices to fully understand this emerging interaction phenomenon.

To examine the opportunities and challenges along the pathway from HCI to HRI, we explore three aspects of interaction - transparency, reflectivity and collaboration-to investigate the role of humanoid factors in the human-robot interaction. This paper reviews recent studies of humanoid robots and discusses the utilization of humanoid factors in practices. Impacts of humanoid factors in transparent, reflective and collaborative interactions are described and examined to strengthen the understanding of humanoid factors in human-robot interaction design. The active understanding 
and learning abilities of the robot are also discussed to highlight differences between human-computer and humanrobot collaborations.

\section{ROBOTIC HUMANOID}

A humanoid robot resembles a human being in many ways. Recent studies in robot-related areas create androids simulating the human both physically and cognitively. Interactions between a human and a robot are also studied in almost all levels, from the fundamental interaction behaviors to the more complex collaboration activities.

Basic activities in HRI have been investigated to enhance the mutual perceptions of human and robot. For example, eye movements that can attract user attention and cues (e.g., glancing, gazing) are used to improve effectiveness of micro events such as hand-over [2], short dialogue [3] and word learning [4]. Head gestures (e.g. nodding, shaking) of both human and robot are investigated as a form of communication cues to direct mutual attention [5, 6]. Hand motion is an important human activity to present human intents. Robots recognizing hand gestures like pointing [7, 8], waving [9] and hand signs $[10,11]$ are leveraged to provide intuitive interaction techniques. All these micro-interactions, which have low physical and mental workload, provide efficient and effective ways to pass information between human and robot. They are not special interactions only work in HRI; most of these gestures have obvious communicative meanings in human-human conversations.

Combining a series of micro-interactions, robots can also collaborate with humans in more complex daily work. In these activates, the humanoid factors inspire people to adopt thinking and behavioral patterns from human-human interaction to the human-robot interaction. For example, the mobility of the robot can assist physically impaired people to perform daily activities such as fetching objects. Similar to asking for help from other people, an intuitive procedure of requesting-to-fetch with a robot might also start from pointing the object with the finger [12]. Robots are also used for social skill training for people with mental disabilities, for example autistic children can practice social activities such as hand shaking and hugging with a robot agent $[13,14]$. When having a robot as a remote conversation tool, it can enhance the sense of presence, and also makes the users to raise etiquette and privacy issues caused by the robot [15]. Similarly, when having a robot in remote education, the embodied interaction expands the dimension of interactions, meanwhile increases the emotionrelated activities like encouragement and embarrassment [16]. Higher level human-robot collaborations have many commons with the social activities amongst human beings. The later directs people's way to interact with a robot, but in some situations has side effects (e.g. privacy issues).

Another application area of humanoid robot can be found in emotional and attentional mediation. Robot's nature of attractiveness make it suitable to address attention and emotion issues, especially for children and elderlies. The adaptive agent for example, is an in-class assistant robot to monitor and capture low-level attention of the students by simulating a real teacher's attention-attracting cues [17]. Also, human's behaviors including body postures and speech are recognized by robots to detect human attention [7, 18]. Comparable cases can be seen in the interaction with social robot. Anthropomorphic robots with the abilities of simulating human facial expressions are developed to offer emotional supports for people who live by themselves [19]. Human factors including facial expressions [20] and speech patterns [21] are utilized by robot to predict human feelings. The design of robots for mental care involves the recognition and simulation of attentional and emotional behaviors of human beings. These applications emphasized the human mental comfort and proposed emotion-friendly interaction techniques.

\section{TWO ASPECTS OF HUMAN COMPUTER INTERACTION}

In contemporary $\mathrm{HCI}$, the participatory role of the user within the development of interactive objects is emphasized to diminish the interaction boundary between the user and the computer which in turn satisfies diverse human values as of emotional and spiritual needs [22]. Transparency and reflectivity are two decisive counterparts in HCI studies; they function differently in different interaction contexts and create embodied and situated, real and reflective experiences.

Transparency in HCI is to reduce the awareness of the interaction mediators. It reflects the expectation that the user focuses more on the job they are doing, while ignores the artefacts they are using [23]. One important goal of HCI research is to understand what is within user's ability to feel the interface is transparent [24]. Besides the artefact itself, the development of the professionalism along the use of the interface is another form of transparency [23]. Modern HCI studies such as ubiquitous computing try to make the computing and the interaction pervasively embedded and situated in the contextual environment and become invisible to the user [25].

Contrary to the transparent interaction, reflectivity emphasize the awareness of the digital artefact, which as a consequence provides unique emotional or aesthetical interaction experience [1]. In addition to the usability of the device, modern reflective designs associate experienceenhancing elements like virtual reality into the interaction to make the experience more real and enjoyable [26], or they try to understand the affection of the user and make the artifact reconfigurable to reflect its user's personality [27].

\section{TRANSPARENT INTERACTION}

Interface transparency is a key factor in human-computer interaction. Interaction devices like the keyboard and the mouse provide easily understandable mappings from the action to the outcome. As a step forward, novel interaction techniques such as NUIs (Natural User Interfaces) seek to utilize gestures and speech to create intuitive and familiar operation methods that require minimal learning [28]. Despite experience-building technologies such as immersive environments that focus on enhancing the feeling with the artifact, control methods are still designed to reduce the workload. In HRI, as humanoid robots are created in the spirit of resembling live creatures, the spontaneous actions can be used to achieve the transparent interaction. 


\section{A. Humanoid Factors in Embodied Interaction}

In the movie WALL.E, when the captain asks Auto (a servant robot wheel) to pause its reporting, he just raises his hand up in front of Auto (Figure 1(a)). A common method to perform a pausing action with a NUI (like Kinect) is by posing a predefined body gesture (Figure 1(b)). Similar operations with traditional WIMP (window, icon, menu and pointer) interfaces are by clicking a button with a pointer (Figure 1(c)).

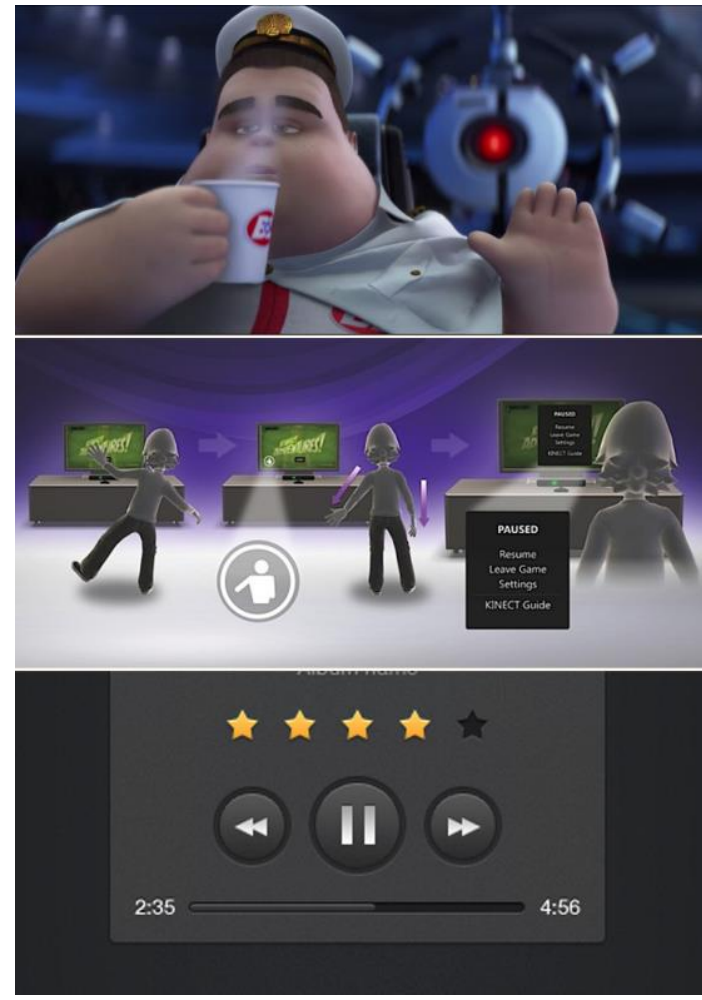

Figure 1. Three different pausing strategies with robot, body motion interface, and traditional WIMP interface.

Instructions using WIMP involve a series of primitive actions. For example, to pause a music player the user has to activate the music player window, move the pointer to the pause button, and click the pause button. With a Kinect, gestures are designated for certain functions, but they still require the user to remember. Since the manner and meaning of these gestures are not always obviously correlated (like the need to extend the left arm at 45 degrees to pause), the humanoid factors provide new opportunities to design transparent operations in which the command can be delivered through the reflexive behaviors triggered by the anthropomorphic stimuli. For example, in asking a user to take an object in WIMP or NUI interfaces, the computer has to show a notification (e.g., textual or linguistic) and the user reads and processes the information before performing the action [29]. With a robot, a similar request can be initiated by mimicking a series of self-objects and then looking at the user (Figure 2) [2]. Another example is if the user denotes an object to a computer, traditional interaction techniques might need the user to touch the object with a pointer, while a robot can recognize hand gestures such as pointing [8]. Through interpreting and performing natural human actions, the robot understands a user's innate social behaviors and makes the interaction transparent. This feature is an example of embodied interaction, with a goal to eliminate distance between intention and action [30]. The operation is in a form relevant to humanoid embodied interaction (which is more similar to interact with real people) rather than manipulating the abstract representations of the computer systems [31].

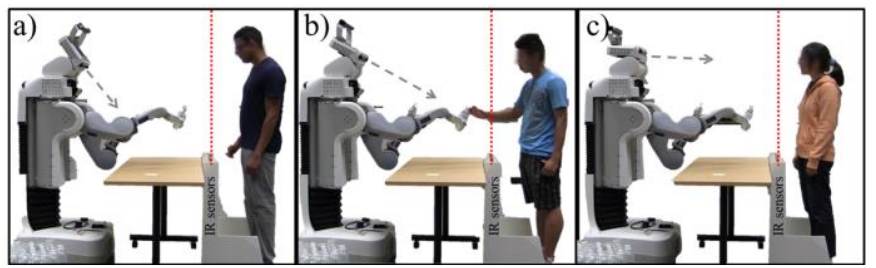

Figure 2. Hand-over process by making eye contact.

Humanoid embodiment of the robot can also increase the transparency of computer supported human communication. Traditional approaches for teleconference with video calls have limitations in transferring environmental information and body motions. It is especially difficult to demonstrate a procedure of operating an object which is far away. The humanoid robot can act as a mediator between remote human-human interactions. With the ability to physically access the object, robot can enhance the embodied feeling with the environment. In the case in remote education, physical interactions through robot not only add another channel of communications, but also bring an interesting and enjoyable aspect to the education process (Figure 3) [16].

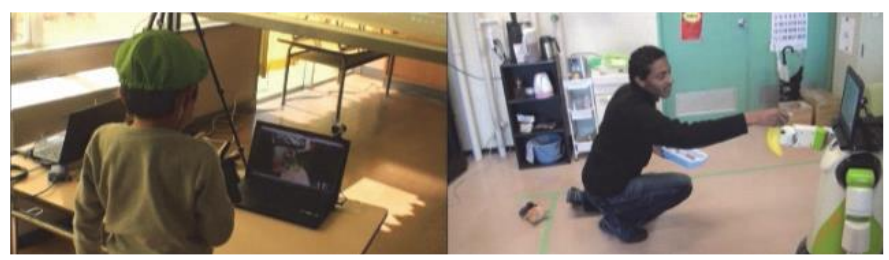

Figure 3. Teleconference for young age education.

On the other hand, challenges brought by the human-like appearances and behaviors should not be ignored. The humanoid factors of the robot are always visible to the user, which will constantly obtain user's attention. Improper configured robot behavior will cause confusion and distraction. For example, eye movements have social meanings to the human. If the eyes of the robot point at some direction not intended by it (perhaps by default), user's attention will unconsciously turn to that object and, then the delay and error might happen [32]. This type of problem results from the fact that user's reflexive reaction to the humanoid robot behavior is usually unconscious. Misinterpretation of the behaviors with strong social meanings is hard to be realized by the user.

\section{B. Humanoid Factors in Situated Interaction}

Situated interaction is a concept that the input of the system is not rigorously determined by the input devices and their prescribed manners of use [33]. The computer should take the entities in the surrounding environment and their dynamic changings into account and make adaptions according to 
different physical and cognitive contexts. Traditional computer software provide configurability to enable the user to adjust the functions according to their needs. However, designing flexible configurations is not straightforward, as understanding what should be configurable is hard and the users might not be able to match their need with the setting options. Novel interaction technologies like smartphones and wearable devices integrate multiple sensors like accelerometer, proximity sensor and GPS to detect the dispositions of the device and make the adapting process transparent to the user [34]. They are successful practices to scaffold situated interaction, because the physical condition of the device and its surrounding environment are well interpreted by the technologies. Robot with more highlevel sensing abilities might expand the understanding of the context from both physical and emotional dimensions. The visual and auditory sensing ability enable humanoids to extract emotional cues from facial expression, utterance and body language [35]. For example, the robot Sage is a museum robot whose behavior varies according to different user moods [36] and Kismet is an expressive robot predicts the identity and status of the speaker through acoustic pattern [21] (Figure 4).

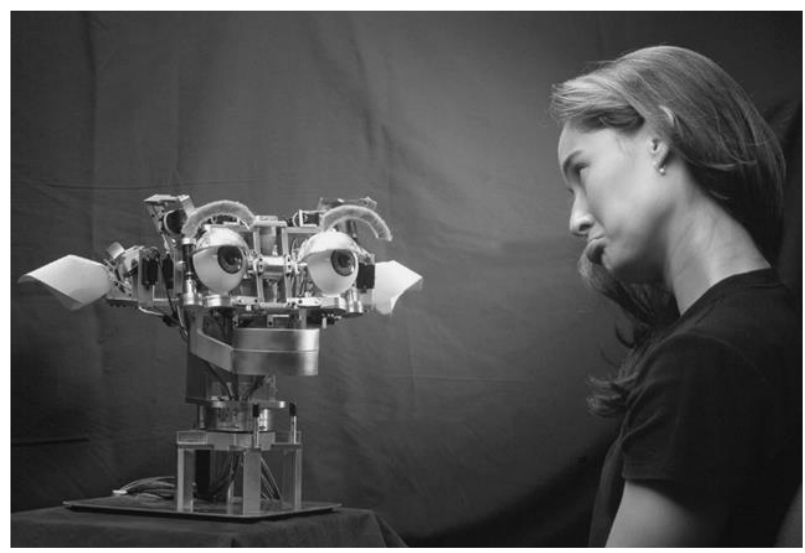

Figure 4. A robot can detect human facial expression.

Besides the sensing abilities, the humanoid factors can be used to design the situated adaption in the multi-user interaction and distributes the message in a transparent way. For example, in a group talking with a robot, the recipient of the information might be only one person. Humanoid elements like eye-contact and head orientation can deliver the information to the pertinent person. Directional actions can gain the attention from the target people, while leaving the irrelevant others uninterrupted [37] (Figure 5).

As the robot is an eye-catching (at least today) and humanlike artefact, people's way of using might not follow the one as it is pre-defined [38]. How to adapt to the contextual changes brought by robot itself, especially the humanoid factors, need to be carefully considered by HRI designers. For instance, potential humanoid-related problems may appear in the humanrobot dialog. Study shows that when talking to a robot, most people spontaneously use natural language ("Could you give me directions to University Center?") rather than the machine language ("locate University Center") [39]. Considering the complexity and fallibility of the human language recognition, it will be a challenge for the robot to understand the user intents.
Incorrect interpretation of the human language will impair the transparency of the interaction.

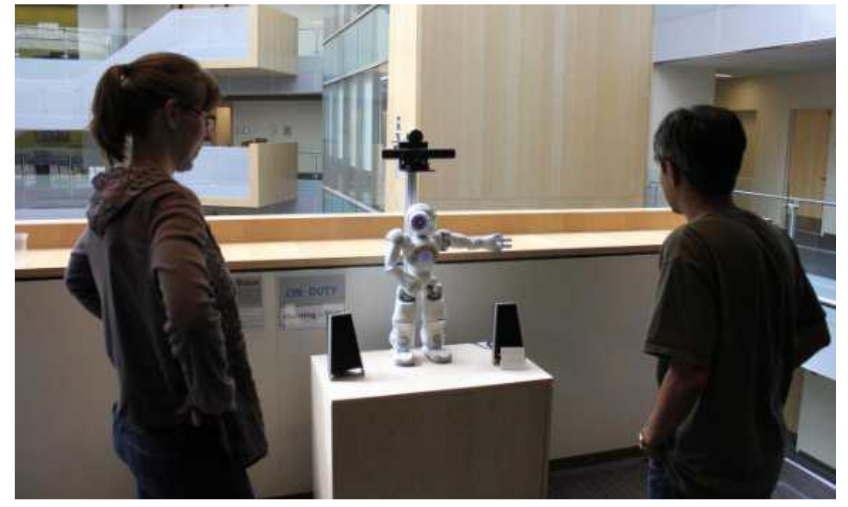

\section{Fig 5. A robot delivers information to pertinent user by looking at the user.}

Another challenge is how to make robots meet primary human values. For example, the vision and memory abilities of a robot mediator might raise user concerns about privacy, surveillance and safety [40]. Moreover, the cultural and regional diversities of the users might result in different perceptions of the humanoid behavior. A study on the robot Geminoid F shows that participants from different areas of the world have different understandings of the same set of android's facial expressions [41]. As the differences in the understanding of the humanoid factors is more subtle comparing to that in the spoken and written language, the design of humanoid behavior needs to be examined and modified to be transparent for people with different cultural backgrounds. The example mentioned above, nodding to confirm, might not be obvious to people from India, where the same gesture is used with an opposite meaning.

\section{REFLECTIVE INTERACTION}

With the evolution of technology, the goal of HCI is no longer limited to improving the efficiency and accuracy of computing devices. The epistemic and emotional values of human-computer interaction have been discovered and developed to provide tools for experience provision, emotion regulation and aesthetic contemplation [26]. Example applications can be seen in virtual reality, affective computing and digital arts. Unlike the claim that the awareness of the interaction mediator should be minimized, these interactions try to furnish epistemic or emotional products through user's reflection on the interaction materials [1]. In HRI, robots such as education robot, training robot and companion robot have been widely used for reflective purposes. Opportunities and challenges of humanoid factors also co-exists in reflective interaction.

\section{A. Artificial Reality}

Using computer to simulate the real world has been studied for decades. Enhancing the feeling of the virtual world generated by the digital technologies is an utmost goal of computer simulation. The real sense created during the interaction not only prolong user's understanding of the real 
world, but also expand human's boundary of imagination. Along the history of $\mathrm{HCI}$, the emerging interaction technologies keep providing and improving the real sense from different channels of perception. In early HCI era, computer graphics is the main source where the sense of reality comes from. By making the virtual world looks and animates visually closer to the real world phenomena, the user obtain a sense of immersion by interacting with the virtual representations. But the virtual world exploration is still constrained by the input and output hardware. Mappings between the motion with the input device like keyboard and mouse are not directly akin to the movements in the virtual environment [42]. The output devices such as computer screen cannot cover the entire visual field of the user either. These drawbacks of the devices undermine the sense of reality and reduce the reflection from the interaction materials. To solve these problems, virtual reality (VR) and augmented reality (AR) technologies are developed to create an immersive interact-able environment which increase the feeling of real presence. Virtual reality utilize projectors or HMDs to display the objects surround the participants, and enhance the sense of being in the virtual environment rather than the place they are physically stay (Figure 6 Left) [43]. Augmented reality differs from VR in that instead of replacing the real world, it allows the participant to see the real objects but with virtual object super-imposed upon or composited with them (Figure 6 Right) [44]. These technologies enhance the feeling with the computer generated material and provide effective and safe methods for experience building and skill development [45].

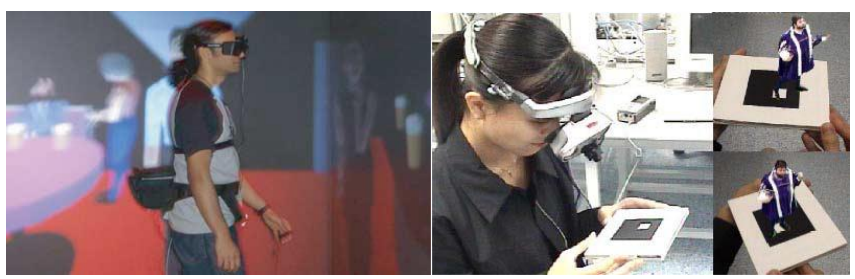

Figure 6. Left: An application using HMD to implement virtual reality. Right: An example application of augmented reality (AR).

The super-real feeling of VR in the virtual environment fundamentally relies on the visual and audio stimuli [46]. The augmented reality supplements reality, providing a sense of coexistence of virtual and real objects [47]. Comparing to these computer graphics based methods of reality simulation, the humanoid robot can also augment the sense of synthetic realism from a perspective of social reality - it constructs a feeling of reality not only with the resembling appearance of the human, but also emotionally real as it is alive.

Feeling as alive is also reflected in the human-robot communication. Human to human communication is a process involves the transmission of cognitive and affective information. Interpersonal sensations such as intimation and inclusion rely on the exchanges of feelings from multiple sensing channels including vision, speech and tactile. [48]. Humanoid factors empower the manlike-androids to simulate real senses from many communication channels, including visual cues, sound, gestures, speech touch, body movements and intelligence [49]. For example, some hyperreal robots with the ability to simulate human facial expressions are created to become an undistinguishable part of future society (Figure 7) [41]. Robot's recognition abilities combined with contact nonlinguistic actions such as kissing, flicking and slapping are also investigated in recent research (Figure 8) [50]. Another application of the socially augmented reality with a robot is in the implementation of assistive technologies, especially for children with autism. Study shows that autistic children show positive behaviors such as making hand and face contacts with the humanoid robot, which benefit them in breaking the social isolation.

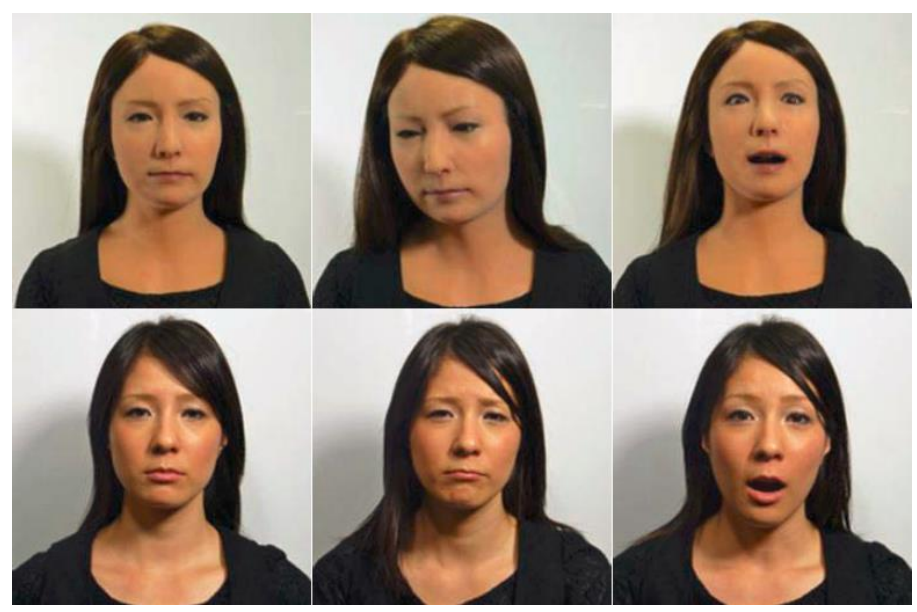

Figure 7. Hyper real robot (top: humanoid robot, bottom: human model).

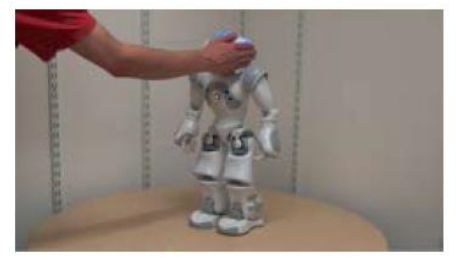

(a) Slap Action

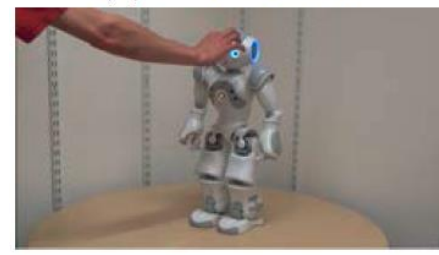

(c) Flick Action

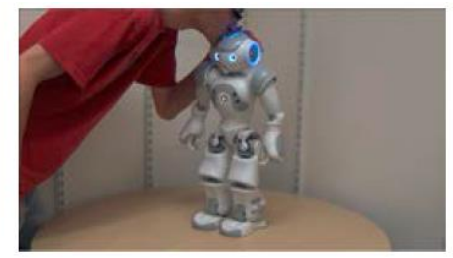

(b) Kiss Action

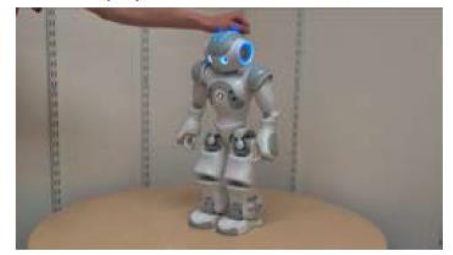

(d) Head Stroke Action
Figure 8. Social activities with a robot.

Comparing to factors such as sound, haptic and engagement which are centric to the reflection of realism in virtual reality [43], the sense of real with a robot also depends on the factors relate to its social abilities. The gap between user's expectation of the man-like response and the actual behavior of the robot impacts the degree of realism in the human-robot interaction. Study suggested that humanoid factors of the robot such as free-will, preferences, emotion and gender will affect human's attitudes towards robots [35]. These human-related factors should be considered in HRI to achieve the real sense in the reflective human-robot interaction. 


\section{B. Personality Scaffolding}

Adding aesthetic elements into the design to endow and reveal personality of the user is another value of reflective interaction. The sense of appropriation of a digital artifact is about increasing its adaptability to the user, empowering and reflecting the user's identity [51]. One pioneer personalizable design which fuses aesthetical elements into a digital device is the iMac computers from Apple (Figure 9). The egg shape and 'flavored' colors (e.g. strawberry red, lime green) of the computer give its user a chance to express their personal care, love and value through a digital device which accompany them every day. Later on, designers of the digital device provide more freedom for the user to personalize the products, from configuring wallpaper of the computer to designing the technology by user themselves. Flexibility in personalization makes the design be taken as a feeling of being moved, and as a consequence the reflection being changed [51].

Modern HCI studies demonstrated that the sense of aesthetic is not limited to the immediate visual impression social, cultural and contextual aspect of the design are all parts of aesthetical reflection [52]. Identifying factors which scaffold user's personal need is pivotal to better user experience via novel technologies. Example findings like the different effects of user relevance and content relevance reveal the importance of personalization reflected by the product and service [53]. Users even assign fundamental personalities to the interaction technologies such as speech control and strongly influenced by these human properties [54].

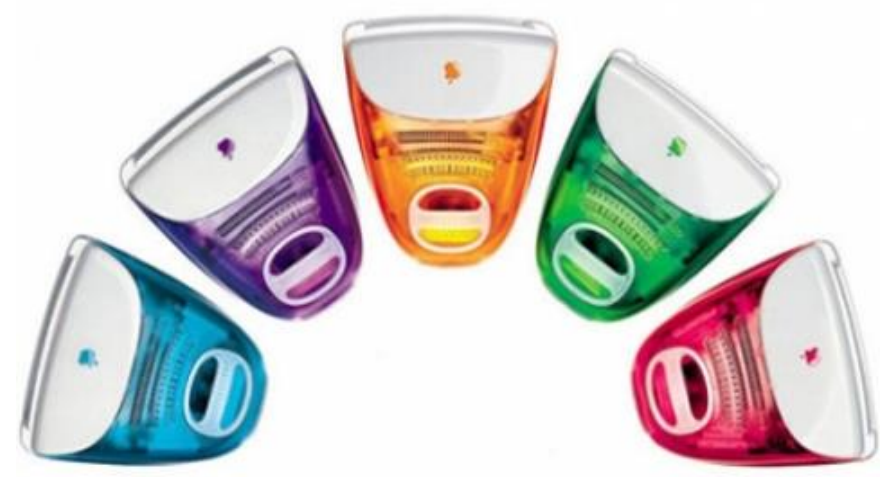

Figure 9. Apple iMac G3 with different color options.

As an extension to the demand of personalization scaffolding technologies, future humanoid robots are always conceived to have its own personalities. Such as JAVIS and Dummy in the movie Iron Man, the former is an intelligent armored suit, highly capable and devoted and the latter is a robot assistant, retarded but funny. In academia, humanoid factors such as emotion, extraversion, agreeableness, conscientiousness and intellect [40] are studied to model the personality of a humanoid robot. However, there is still a debate about whether the personality of a robot should or should not match the one of its human user [55]. Some experiment shows that the extraversion-introversion personality matching is preferred by participants who use robot as an assistive technology [56]. But others also pointed out that if the robot performs better at self-relevant tasks, users will be threatened by the robot [57]. People's opinion towards the reflection of personality by a highly intelligent artifacts might be heavily impacted by cultural background and psychological condition. How to design a personality scaffolding robot still waits to be discovered by HRI practitioners.

\section{HUMAN ROBOT COLLABORATION}

Collaborating with a robot in a task is a process influenced by factors from both user's experience with the computer and human-human collaboration practices. Making the humanrobot collaboration merely transparent or reflective is a misinterpretation of the nature of the future high intelligent and human-like android. Designing a robot which support safe, seamless and effective collaboration with a human user requires a complete understanding of the abilities a robot should have, including perceiving and understanding the environment, making a plan, and learning from experience [58]. The human-robot collaboration differs from traditional HCI in that a robot should actively aware the mental and physical intentions of its human user and response his activities in an anthropomorphic way.

Most traditional computer-based systems do not actively comprehend human users. They passively receive instructions from the user and process their requirements in established ways. Even in modern CSCW theories, the role of computing artefacts still largely lies in acting as a material to be constructed, applied and adapted in people's collaborative practice [33]. Humanoid robot however, as an intelligent agent with social ability and behavior, make the human users perceive them as being able to actively guide the interaction. It is expected that by acquiring a small advice (clapping hand, short whistle) from the human user, a robot can perform the task automatically [59]. As such, how to actively understand human user's intents is essential for a robot. Related studies probed using short dialogues to instruct the robot [59]. Devices which detect physiological signals such as heart rate and skin conductance are also utilized to monitor human's mental activities [60]. To meet human's expectation of the understanding ability during the human-robot collaboration, future robot should be able to associate those multi-channel social cues to actively predict human intents.

Learning and imitation is another important humanoid factor of robotic intelligence. The more a robot knows about a user's behavioral patterns, the better it can understand the user. In recent studies, abilities and information to be learned by a robot include the spatial information [61], human demonstration [62] and navigation [63]. By enabling a robot to remember the information and activities generated during the collaboration, a robot keeps track of the interaction patterns of the human and be able to repeat complex activities triggered by a simple user action.

Human-robot collaboration is a dynamic integration of both transparent and reflective interactions. As the humanoid factors of the robot lie in the fact that it is an intelligent machine while bearing human-related factors, the design of collaborative humanoid robot should both consider it as tool, which can finish the task without much human efforts, and meanwhile 
provide cognitive scaffoldings such as active comprehension, learning and imitation.

\section{DISCUSSION AND CONCLUSION}

Humanoid factors of robots make interaction with them different than with other computing machines. By comparing and contrasting technologies of robot and other interaction methods, this paper reviews opportunities and challenges of humanoid factors with respect to traditional HCI design. The influence and inspiration gleaned from robotic humanoid factors are explored with respect to the transparency and reflectivity of the interaction. Findings in this work aim to shed light on design knowledge for future human-robot interaction.

Humans' innate ability to understand social activities is meaningful to achieve intuitive and natural interaction with a robot. In most classic interaction techniques with keyboard and mouse, the seamlessness of the interaction relies on people's professional skills of the input-output mapping. Novel interaction devices makes the mapping easier to build, but most of which still need the user to get used to the interaction paradigm. Simulating a social behavior can make the perception of the robot-generated operation embodied and situated to human users. By interpreting a human's reflexive social behavior, interactions to send and deliver information with a human-like robot can be transparent.

Traditional reflective interaction can impress the user by creating virtual reality or emotionally ensouled artifacts. The humanoid factor provides a new pathway to enhance the artificial reality from a social perspective-humanoid robots can reflect the sense of real not only visually, acoustically, and tactilely, but also emotionally, affectively and cognitively. Furthermore, with increasing interests on the studies of the robot's personalities, though their reflection on the human's personality is still unclear, future HRI designs should take the emotional and spiritual traits of robot into consideration, to best fit user's emotional and aesthetical need.

To support human-robot collaboration, the transparency and reflectivity of the interaction should be considered comprehensively, in terms of social behaviors such as active understanding and learning. The human-like senses and cognitive patterns will help to achieve smooth and effective human-robot collaboration.

\section{FUTURE WORK}

Will humanoid robots eventually become a real member in our life as the way we are using our smart phones or refrigerators today that we feel so comfortable and intimate with? Since the robot technology and interaction methods keep growing and expanding, it is impossible to answer the question only with existing HCI knowledge. This paper identifies humanoid factors as a key design element in human-robot interaction, but it is only a starting point. A more complete answer relies on the imagination and ingeniousness of robot designers to constantly define and redefine the relationship between human and artefacts. The path from HCI to HRI is a long one, where previous HCI knowledge should be reused critically, and new HRI theories need to be discovered.

\section{REFERENCES}

[1] J. D. Bolter and D. Gromala, "Transparency and Reflectivity: Digital Art and the Aesthetics of Interface Design," Aesthetic computing, p. 369, 2006.

[2] A. Moon, D. M. Troniak, B. Gleeson, M. K. Pan, M. Zeng, B. A. Blumer, et al., "Meet me where i'm gazing: how shared attention gaze affects human-robot handover timing," in Proceedings of the 2014 ACM/IEEE international conference on Human-robot interaction, 2014, pp. 334-341.

[3] C. Liu, C. T. Ishi, H. Ishiguro, and N. Hagita, "Generation of nodding, head tilting and eye gazing for human-robot dialogue interaction," in Human-Robot Interaction (HRI), 2012 7th ACM/IEEE International Conference on, 2012, pp. 285-292.

[4] C. Yu, M. Scheutz, and P. Schermerhorn, "Investigating multimodal real-time patterns of joint attention in an hri word learning task," in Proceedings of the 5th ACM/IEEE international conference on Humanrobot interaction, 2010, pp. 309-316.

[5] A. Yamazaki, K. Yamazaki, Y. Kuno, M. Burdelski, M. Kawashima, and H. Kuzuoka, "Precision timing in human-robot interaction: coordination of head movement and utterance," in Proceedings of the SIGCHI Conference on Human Factors in Computing Systems, 2008, pp. 131-140.

[6] C. L. Sidner, C. Lee, L.-P. Morency, and C. Forlines, "The effect of head-nod recognition in human-robot conversation," in Proceedings of the 1st ACM SIGCHI/SIGART conference on Human-robot interaction, 2006, pp. 290-296.

[7] K. Nickel and R. Stiefelhagen, "Visual recognition of pointing gestures for human-robot interaction," Image and Vision Computing, vol. 25, pp. 1875-1884, 2007.

[8] M. Van den Bergh, D. Carton, R. De Nijs, N. Mitsou, C. Landsiedel, K. Kuehnlenz, et al., "Real-time 3D hand gesture interaction with a robot for understanding directions from humans," in RO-MAN, 2011 IEEE, 2011, pp. 357-362.

[9] C. L. Nehaniv, K. Dautenhahn, J. Kubacki, M. Haegele, C. Parlitz, and R. Alami, "A methodological approach relating the classification of gesture to identification of human intent in the context of human-robot interaction," in Robot and Human Interactive Communication, 2005. ROMAN 2005. IEEE International Workshop on, 2005, pp. 371-377.

[10] X. Yin and M. Xie, "Finger identification and hand posture recognition for human-robot interaction," Image and Vision Computing, vol. 25, pp. 1291-1300, 2007.

[11] M. Correa, J. Ruiz-del-Solar, R. Verschae, J. Lee-Ferng, and N. Castillo, "Real-time hand gesture recognition for human robot interaction," in RoboCup 2009: Robot Soccer World Cup XIII, ed: Springer, 2010, pp. 46-57.

[12] H. Nguyen, C. Anderson, A. Trevor, A. Jain, Z. Xu, and C. C. Kemp, "El-e: An assistive robot that fetches objects from flat surfaces," in Robotic helpers, int. conf. on human-robot interaction, 2008.

[13] B. Robins, K. Dautenhahn, and P. Dickerson, "From isolation to communication: a case study evaluation of robot assisted play for children with autism with a minimally expressive humanoid robot," in Advances in Computer-Human Interactions, 2009. ACHI'09. Second International Conferences on, 2009, pp. 205-211.

[14] W. D. Stiehl, C. Breazeal, K.-H. Han, J. Lieberman, L. Lalla, A. Maymin, et al., "The huggable: a therapeutic robotic companion for relational, affective touch," in ACM SIGGRAPH 2006 emerging technologies, 2006, p. 15.

[15] J. M. Beer and L. Takayama, "Mobile remote presence systems for older adults: acceptance, benefits, and concerns," in Proceedings of the 6th international conference on Human-robot interaction, 2011, pp. 19-26.

[16] F. Tanaka, T. Takahashi, S. Matsuzoe, N. Tazawa, and M. Morita, "Telepresence robot helps children in communicating with teachers who speak a different language," in Proceedings of the 2014 ACM/IEEE international conference on Human-robot interaction, 2014, pp. 399406.

[17] D. Szafir and B. Mutlu, "Pay attention!: designing adaptive agents that monitor and improve user engagement," in Proceedings of the SIGCHI Conference on Human Factors in Computing Systems, 2012, pp. 11-20.

[18] B. Schauerte and G. A. Fink, "Focusing computational visual attention in multi-modal human-robot interaction," in International Conference 
on Multimodal Interfaces and the Workshop on Machine Learning for Multimodal Interaction, 2010, p. 6.

[19] C. Breazeal, "Emotion and sociable humanoid robots," International Journal of Human-Computer Studies, vol. 59, pp. 119-155, 2003.

[20] G. Pioggia, R. Igliozzi, M. Ferro, A. Ahluwalia, F. Muratori, and D. De Rossi, "An android for enhancing social skills and emotion recognition in people with autism," Neural Systems and Rehabilitation Engineering, IEEE Transactions on, vol. 13, pp. 507-515, 2005.

[21] C. Breazeal and L. Aryananda, "Recognition of affective communicative intent in robot-directed speech," Autonomous robots, vol. 12, pp. 83104, 2002.

[22] S. Bødker, "When second wave HCI meets third wave challenges," in Proceedings of the 4th Nordic conference on Human-computer interaction: changing roles, 2006, pp. 1-8.

[23] J. E. Bardram and O. W. Bertelsen, "Supporting the development of transparent interaction," in Human-Computer Interaction, ed: Springer, 1995, pp. 79-90.

[24] G. W. Strong, "New directions in human-computer interaction: education, research, and practice," interactions, vol. 2, pp. 69-81, 1995.

[25] G. D. Abowd, "Software engineering issues for ubiquitous computing," in Software Engineering, 1999. Proceedings of the 1999 International Conference on, 1999, pp. 75-84.

[26] L. K. Hansen, "Contemplative interaction: alternating between immersion and reflection," in Proceedings of the 4th decennial conference on Critical computing: between sense and sensibility, 2005, pp. 125-128.

[27] R. Cowie, E. Douglas-Cowie, N. Tsapatsoulis, G. Votsis, S. Kollias, W. Fellenz, et al., "Emotion recognition in human-computer interaction," Signal Processing Magazine, IEEE, vol. 18, pp. 32-80, 2001.

[28] D. Wigdor and D. Wixon, Brave NUI world: designing natural user interfaces for touch and gesture: Elsevier, 2011.

[29] E. Horvitz, C. Kadie, T. Paek, and D. Hovel, "Models of attention in computing and communication: from principles to applications," Communications of the ACM, vol. 46, pp. 52-59, 2003.

[30] T. Starner, "Project glass: An extension of the self," Pervasive Computing, IEEE, vol. 12, pp. 14-16, 2013.

[31] P. Dourish, "Seeking a foundation for context-aware computing," Human-Computer Interaction, vol. 16, pp. 229-241, 2001.

[32] H. Kuzuoka, K. Yamazaki, A. Yamazaki, J. i. Kosaka, Y. Suga, and C. Heath, "Dual ecologies of robot as communication media: thoughts on coordinating orientations and projectability," in Proceedings of the SIGCHI conference on Human factors in computing systems, 2004, pp. 183-190.

[33] K. Schmidt, Cooperative work and coordinative practices: Springer, 2011.

[34] K. Lyons and H. Profita, "The Multiple Dispositions of On-Body and Wearable Devices," Pervasive Computing, IEEE, vol. 13, pp. 24-31, 2014.

[35] T. Fong, I. Nourbakhsh, and K. Dautenhahn, "A survey of socially interactive robots," Robotics and autonomous systems, vol. 42, pp. 143166, 2003.

[36] I. R. Nourbakhsh, J. Bobenage, S. Grange, R. Lutz, R. Meyer, and A. Soto, "An affective mobile robot educator with a full-time job," Artificial Intelligence, vol. 114, pp. 95-124, 1999.

[37] D. Bohus and E. Horvitz, "Managing Human-Robot Engagement with Forecasts and... um... Hesitations," in Proceedings of the 16th International Conference on Multimodal Interaction, 2014, pp. 2-9.

[38] L. A. Suchman, "Office procedure as practical action: models of work and system design," ACM Transactions on Information Systems (TOIS), vol. 1, pp. 320-328, 1983.

[39] M. K. Lee and M. Makatchev, "How do people talk with a robot?: an analysis of human-robot dialogues in the real world," in $\mathrm{CHI}^{\prime} \mathrm{OS}$ Extended Abstracts on Human Factors in Computing Systems, 2009, pp. 3769-3774.

[40] D. S. Syrdal, K. Dautenhahn, S. N. Woods, M. L. Walters, and K. L. Koay, "Looking Good? Appearance Preferences and Robot Personality Inferences at Zero Acquaintance," in AAAI Spring Symposium: Multidisciplinary Collaboration for Socially Assistive Robotics, 2007, pp. 86-92.
[41] C. Becker-Asano and $H$. Ishiguro, "Evaluating facial displays of emotion for the android robot Geminoid F," in Affective Computational Intelligence (WACI), 2011 IEEE Workshop on, 2011, pp. 1-8.

[42] J. J. Laviola, "Bringing VR and spatial 3D interaction to the masses through video games," Computer Graphics and Applications, IEEE, vol. 28, pp. 10-15, 2008.

[43] M. V. Sanchez-Vives and M. Slater, "From presence to consciousness through virtual reality," Nature Reviews Neuroscience, vol. 6, pp. 332339, 2005.

[44] R. T. Azuma, "A survey of augmented reality," Presence, vol. 6, pp. 355-385, 1997.

[45] S. A. Green, M. Billinghurst, X. Chen, and G. Chase, "Human-robot collaboration: A literature review and augmented reality approach in design," 2008.

[46] R. Patterson, M. D. Winterbottom, and B. J. Pierce, "Perceptual issues in the use of head-mounted visual displays," Human Factors: The Journal of the Human Factors and Ergonomics Society, vol. 48, pp. 555-573, 2006.

[47] P. Mistry and P. Maes, "SixthSense: a wearable gestural interface," in ACM SIGGRAPH ASIA 2009 Sketches, 2009, p. 11.

[48] F. Biocca and M. R. Levy, Communication in the age of virtual reality: Routledge, 2013

[49] H. R. Lee and S. Sabanović, "Culturally variable preferences for robot design and use in South Korea, Turkey, and the United States," in Proceedings of the 2014 ACM/IEEE international conference on Human-robot interaction, 2014, pp. 17-24.

[50] R. Read and T. Belpaeme, "Situational context directs how people affectively interpret robotic non-linguistic utterances," in Proceedings of the 2014 ACM/IEEE international conference on Human-robot interaction, 2014, pp. 41-48.

[51] P. Turner, "How We Cope with Digital Technology," Synthesis Lectures on Human-Centered Informatics, vol. 6, pp. 1-109, 2013.

[52] M. G. Petersen, O. S. Iversen, P. G. Krogh, and M. Ludvigsen, "Aesthetic Interaction: a pragmatist's aesthetics of interactive systems," in Proceedings of the 5th conference on Designing interactive systems: processes, practices, methods, and techniques, 2004, pp. 269-276.

[53] M. de Sa, V. Navalpakkam, and E. F. Churchill, "Mobile advertising: evaluating the effects of animation, user and content relevance," in Proceedings of the SIGCHI Conference on Human Factors in Computing Systems, 2013, pp. 2487-2496.

[54] C. Nass and K. M. Lee, "Does computer-generated speech manifest personality? An experimental test of similarity-attraction," in Proceedings of the SIGCHI conference on Human Factors in Computing Systems, 2000, pp. 329-336.

[55] S. Woods, K. Dautenhahn, C. Kaouri, R. Boekhorst, and K. L. Koay, "Is this robot like me? Links between human and robot personality traits," in Humanoid Robots, 2005 5th IEEE-RAS International Conference on, 2005, pp. 375-380.

[56] A. Tapus and M. J. Matarić, "User personality matching with a handsoff robot for post-stroke rehabilitation therapy," in Experimental robotics, 2008, pp. 165-175.

[57] H. Kamide, K. Kawabe, S. Shigemi, and T. Arai, "Social Comparison between the Self and a Humanoid," in Social Robotics, ed: Springer, 2013, pp. 190-198.

[58] A. Bauer, D. Wollherr, and M. Buss, "Human-robot collaboration: a survey," International Journal of Humanoid Robotics, vol. 5, pp. 47-66, 2008

[59] T. Fong, C. Thorpe, and C. Baur, "Collaboration, dialogue, human-robot interaction," in Robotics Research, ed: Springer, 2003, pp. 255-266.

[60] P. Rani, N. Sarkar, C. A. Smith, and L. D. Kirby, "Anxiety detecting robotic system-towards implicit human-robot collaboration," Robotica, vol. 22, pp. 85-95, 2004.

[61] B. Kuipers and Y.-T. Byun, "A Robust, Qualitative Method for Robot Spatial Learning," in AAAI, 1988, pp. 774-779.

[62] B. D. Argall, S. Chernova, M. Veloso, and B. Browning, "A survey of robot learning from demonstration," Robotics and autonomous systems, vol. 57, pp. 469-483, 2009.

[63] G. N. DeSouza and A. C. Kak, "Vision for mobile robot navigation: A survey," Pattern Analysis and Machine Intelligence, IEEE Transactions on, vol. 24, pp. 237-267, 2002. 